\title{
THE LOW FREQUENCY VARIABILITY OF EXTRAGALACTIC RADIO SOURCES: A RELATIVISTIC EFFECT OR GALACTIC SCINTILLATION ?
}

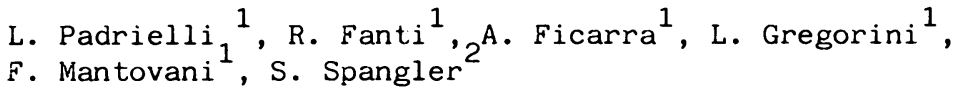 \\ 1. Istitıto di Radioastronomia, Bologna, Italy \\ 2. Dep. of Physics and Astronomy, University of Iowa, USA
}

Results obtained on the Low Frequency Variability (LFV) phenomenon, by
means of combined multifrequency observations of 50 sources, on a period
of more than ten years on a frequency grid of $0.4,2.3,4.8,8.0$, and
$14.4 \mathrm{GHz}$ and two epoch VLBI observations at $18 \mathrm{~cm}$ can be summarized as
follows: 1. $15-20 \%$ of variables appear to have variations consisting either of quasi-simultaneous outbursts at all frequencies or of bursts which drift to lower frequencies with time and decreasing amplitude. In our sample, we find five good cases: 3C 120, 0605-085, 1510-089, 3C 345, BL Lac. Three of these are famous superluminals; the other two show significant structural changes between our $18 \mathrm{~cm}$ VLBI measurements. The corresponding expansion rate for these five sources is in agreement with the $\gamma^{\prime} s$ derived from LFV with the usual causality arguments. For the sources of this class, the observations are therefore in agreement with models that explain the phenomenon of the variability as synchrotron emission of relativistic electrons beamed in a direction close to the line of sight.

2. $35 \%$ of variables show only low frequency $(<1 \mathrm{GHz}$ ) variability and little or no intermediate high frequency variations. In DA 406, prototype of the category, no superluminal motions have been observed, even if the resolution of our VLBI observations should allow the detection of the structural change expected on the basis of intrinsic LFV. In this case we do not find direct evidence of relativistic motions associated with the LFV and the process is most easily explained if the variations are extrinsic (propagation effects through the interstellar medium as the slow refractive scintillation).

3. The remaining $40-45 \%$ of variables show uncorrelated high ( $>5 \mathrm{GHz}$ ) and low frequency variability with a minimum of activity at the intermediate frequencies. The explanation of the phenomenon is less clear. It could be attributed to intrinsic (superluminal) variations at high frequencies, coexisting with unrelated processes at low frequencies.

It is quite likely that both intrinsic and extrinsic processes are at work in the LFV. 
We have accepted as "bona fide" intrinsic variables the five sources of the first category; we ask whether the remaining sources are all explained by the scintillation model and what parameters are required by the interstellar medium.

The variability measurements are characterized by two parameters: the scintillation index $(m=r . m . s . / a v e r a g e ~ f l u x)$, and the time scale $(t)$. We statistically derived these parameters for the 50 LFV sources monitored at $0.4 \mathrm{GHz}$, by means of structure function and autocorrelation function. We have found that $30 \%$ and perhaps as many as $50 \%$ of the sources show two distinct time scales. The rms flux variability associated with each of the two time scales has been evaluated and two variability indices $m$ short and $\mathrm{m}$ long have been computed.

In the light of the predictions of a slow refractive scintillation model (Rickett, 1986, Ap. J, 307, 564), a relation has been derived between the observable quantities $(m, t$, and galactic latitude (b) of the source) and the properties of the medium.

$\mathrm{m} t(\sin \mathrm{b})^{3 / 2}=\mathrm{f}($ medium $)$

The quantity $m^{*} t$ is, to first order, independent of source characteristics.

The distribution of the $m^{*} t$ associated with the shorter tirie scales follows the expected $\sin b^{-3 / 2}$ law with a large scatter. Noticeable exceptions are present: DA 406 and $1117+14$ vary too slowly or too much. Furthermore two sources with low galactic latitude in the direction of the galactic anticenter have very small $m^{*} t$. This latter anomaly is not unexpected, owing to the finite size of the Galaxy.

The products $m^{*} t$ associated with the longer time scales do not show a clear correlation with the galactic latituce.

It could be argued that the large scatter results from merging all sources with the same absolute magnitude of b; the characteristics of the medium could be different for positive or negative $b$ and for different galactic longitude. To circumvent this problem we have considered groups of sources near each other within a few degrees; the radiation of the sources should pass through a medium with similar characteristics and $m^{*} t$ should be similar. The data only weakly support this hypothesis, because the scatter does not decrease substantially. In particular DA 406 and $3 C 345,8 \mathrm{deg}$. close in the sky, show definitely different variability behaviour, even if their low frequency VLBI structures are very similar on a scale of 10 mas.

Finally we want to remark that the variability parameters related to propagation effects show $m^{*} t$ values less than those quoted by Rickett (1986). This requires some modification of the medium model.

\section{CONCLUSION}

$15-20 \%$ of LFV sources give indications of intrinsic variability. A component of LFV can be due to propagation effects through the Galaxy. However several sources show a longer time scale variability, whose nature is not understood: they could define a new class of intrinsic variables. 